\title{
Departmental Compliance with Enoxaparin Prescribing in Orthopaedic Wards of the RAH
}

\section{Robert Tyler* and Laura Sayers}

Royal Alexandra Hospital, Corsebar Road, Paisley, PA2 9PN, Scotland

\begin{abstract}
Paisley's Trauma and Orthopaedics Department prescribes enoxaparin for prophylaxis of thromboembolic events in both elective and trauma patients. It is pertinent that this is prescribed at the correct dose, adjusted for weight and renal function to ensure that prophylaxis is sufficient without increasing post-operative haemorrhage risk. Patients with a weight of less than $50 \mathrm{kgs}$ or a creatinine clearance of less than $30 \mathrm{mls} /$ minute should be prescribed a half dose of enoxaparin.
\end{abstract}

Methods: Two periods of evaluation were undertaken involving a retrospective review of trauma and elective patients. The first loop examined 100 sets of case notes; the second loop examined 50 completing a full audit cycle.

The following was extracted for each patient:

1) Admission dose of enoxaparin

2) Weight on admission

3) Creatinine clearance on admission

For each patient, it was concluded whether that dose was appropriate for the patient using the weight and creatinine clearance.

\section{Results cycle one}

- $11 \%$ of prescriptions incorrect

- $7 \%$ creatinine clearance incorrectly calculated

- $4 \%$ weight $<50 \mathrm{~kg}$

\section{Intervention:}

1) Designed original posters highlighting the need for dose adjustment in patients with low weight/impaired renal function

2) Displayed posters in clinical areas

3) Incorporated poster into new staff induction packs

4) Awareness amongst current staff rose through discussion and presentation of audit findings Cycle

Two

- $4 \%$ of prescriptions incorrect

- Overall reduction in prescribing errors by of $63 \%$

Discussion: The two cycles showed that prescribing error.

Is a significant problem and that patient safety was a concern, particularly on surgical wards where haemorrhage risk is increased. Successful changes were introduced which resulted in a noticeable improvement in prescribing practice.

\section{Introduction}

Enoxaparin - trade name; clexane, is a commonly prescribed drug in all wards of a hospital, especially in surgical wards such as orthopaedics. In both elective and trauma units it is prescribed for prevention of deep vein thrombosis in patients who have undergone or are about to undergo corrective surgery. It works by promoting the action of antithrombin III, therefore inhibiting Factor Xa, reducing the production of thrombin and therefore fibrin clot formation. The British National Formulary (BNF) states that enoxaparin should be prescribed as a once daily subcutaneous dose of $40 \mathrm{mg}$. This is unless the patient has a weight of less than $50 \mathrm{~kg}$ or a creatinine clearance of less than $30 \mathrm{mls}$ per minute. If either of these holds true, then 20 $\mathrm{mg}$ of enoxaparin should be prescribed. It has long been thought that eGFR will suffice instead of calculating the patient's creatinine clearance. It is, however, clearly stated in the BNF that 'for potentially toxic drugs' or patient's 'at extremes of weight [1], the creatinine clearance should be calculated. Problems caused by prescribing errors

*Corresponding author: Robert Tyler, Royal Alexandra Hospital, Corsebar Road, Paisley, PA2 9PN, Scotland, E-mail: tyler836@hotmail.com

Received March 16, 2013; Accepted April 29, 2013; Published May 07, 2013

Citation: Tyler R, Sayers L (2013) Departmental Compliance with Enoxaparin Prescribing in Orthopaedic Wards of the RAH. Surgery S12: 015. doi:10.4172/21611076.S12-015

Copyright: ( 2013 Tyler R, et al. This is an open-access article distributed under the terms of the Creative Commons Attribution License, which permits unrestricted use, distribution, and reproduction in any medium, provided the original author and source are credited. 
in thromobo prohylaxis are well documented; 'iatrogenic haemorrhage is not uncommon in those with chronic kidney disease or a low BMI [2]. In low weight women ( $<45 \mathrm{kgs})$ and low weight men $(<57 \mathrm{kgs})$ an increase in enoxaparin exposure has been observed. Furthermore, in patients with severe renal impairment $(\mathrm{CrCl}<30 \mathrm{ml} / \mathrm{min})$ the steady state is significantly increased by an average of $65 \%$ [2]. This illustrates how serious incorrect prescribing can become, especially in orthopaedic wards, where the very nature of the injuries sustained and the operation itself, promote blood loss. A scenario to highlight the perils of incorrect prescribing goes as follows. The on-call junior doctor is called to the emergency department to clerk-in a 77-year old lady with a fractured neck of femur. She is known to have a long history of chronic kidney disease. Blood tests are taken in the emergency department and the results are available two hours later. The junior doctor prescribes $40 \mathrm{mg}$ enoxaparin on the basis she weighs more than $50 \mathrm{~kg}$ but fails to check either old biochemistry data or the blood results when they become available. The creatinine comes back later at 289 and is not used as part of the prescription calculation. This would leave the creatinine clearance at 22 and mean a $20 \mathrm{mg}$ dose. She remains on the ward for three weeks after acquiring nosocomial pneumonia and is then discharged. Iatrogenic haemorrhage is something we want to avoid. When working in the orthopaedic unit it became apparent that prescribing errors were being made and the above scenario was indeed occurring, with patients receiving a higher dose of enoxaparin than needed. There is very little in the literature that addresses the doses of low molecular weight heparin and associated risks. The bulk of the literature concerns whether patients receive thrombo prophylaxis after being risk stratified. The aim of this retrospective study was to audit the prescribing of enoxaparin, highlight any concerns, institute changes if necessary and then complete the audit-loop one month later.

\section{Method}

We retrospectively reviewed 100 patients, from both elective and trauma units over a period of one month. The inclusion criteria stated that patients were admitted to either the trauma or elective ward within two weeks from the study beginning and they were not already being prescribed LMWH prior to admission. With this group of

Patients we would identify only those who had been prescribed LMWH by the junior doctors in the department. The admission dose of enoxaparin, height and weight and creatinine clearance was recorded for each patient. This gave us both parameters needed to assess whether enoxaparin had been prescribed correctly. BNF and SIGN guidelines 122 were used as our clinical standards. When a prescription was calculated incorrectly, the specific reason for this was documented. Intervention

This was achieved in the following ways:

1. We raised awareness with current staff by discussing the data with current junior doctors at the morning trauma meeting.

2. We asked the clinician in charge of the introductory meeting for the new cohort of junior doctors to incorporate our data into his lecture.

3. We put up posters highlighting the results of our data in all the relevant clinical areas of the orthopaedics department. These were the wards, the plaster room, the fracture clinic, the preassessment clinic and the trauma theatre.

\section{Re-audit}

After the above measures were taken, a re-audit was carried out two weeks later, with a smaller cohort of fifty patients. This was done in exactly the same way, taking us two weeks to collect and collate the data.

\section{Results}

\section{$1^{\text {st }}$ audit loop}

Of the 100 prescriptions retrospectively audited, 11/100 was found to have errors.

The reasons for these 11 errors are documented below:

1. Creatinine Clearance $<30 \mathrm{mls} /$ minute and full dose enoxaparin prescribed: 7 errors (A)

2. Weight $<50 \mathrm{~kg}$ and full dose of enoxaparin prescribed: 4 errors. (B)

\section{Re-audit}

The results highlighted two errors in enoxaparin prescribing:

1. Creatinine Clearance $<30 \mathrm{mls} /$ minute and full dose enoxaparin prescribed: 1 error (A)

2. Weight $>50 \mathrm{~kg}$, creatinine clearance $>30$ and half dose enoxaparin prescribed. 1 error (C)

In the first audit cycle, the prescription error rate is at $11 \%$. This is reduced in the re-audit to $4 \%$.

\section{Discussion}

This discussion aims to look at some of the areas where errors were being made in relation to enoxaparin prescribing and how we managed to find ways around these, so as to ensure safe prescribing. The above errors can be complicated to comprehend so the exact mechanism in which they occur are further discussed here.

To break it down, there are three main errors that could occur.

A. The creatinine clearance is $<30 \mathrm{mls} /$ minute but a full dose of $40 \mathrm{mg}$ is prescribed. This happens because junior doctors are calculating incorrectly or simply unaware that knowing renal function is integral to dose calculation.

B. When a dose is being prescribed, the prescriber takes no notice of the patient's weight and prescribes $40 \mathrm{mg}$ when they weigh less than $50 \mathrm{~kg}$. This should mean a reduced dose of $20 \mathrm{mg}$.

C. Weight $>50 \mathrm{kgs}+$ Creatinine clearance $>30 \mathrm{mls} /$ minute - half dose received. This may reflect the zeal at which we tried to implement the changes to make sure doses weren't too high, with a prescriber being too careful in their enoxaparin dose. It is, however, only one result out of fifty and erroneous in many respects.

It must be highlighted that none of the above errors were made due to the dose being too low. All errors were due to the dose being too high. As the two primary reasons for incorrect prescribing had been identified, it was then much easier to attempt to make changes to current practice in the form of an intervention as is commonly in done in a full audit loop. Following this intervention, the above results highlighted a marked improvement in the departments prescribing. The error rate had dropped from $11 \%$ to $4 \%$. The original scenario is not altogether unlikely, and the various parts of this story were found to occur on more than one occasion when looking into where mistakes were made. The two pieces of information learnt from this were: 
1. Blood samples that are taken in the acute setting must be chased up when the patient is then admitted.

2. When prescribing enoxaparin 'blind' (i.e. without blood results) use the most recent blood samples available on the system or wait until later when this information becomes available. The second reason that errors were made was much easier to appreciate. Doctors were prescribing the full dose of enoxaparin when the patient weighed less than $50 \mathrm{~kg}$. The blame here can somewhat be shifted from the prescriber as sometimes the weight has not become available. A good example of this is patients who have dementia will not be able to disclose this information and it is therefore forgotten about. To compound this problem, these are the exact patients who are underweight and need lower doses of enoxaparin. To prevent this from happening, nursing staff must be reminded to weigh patients at the earliest opportunity and doctors must check weights every time they prescribe enoxaparin. In the cases of poly trauma where patients cannot be weighed, family members or nursing home staff can assist, or at worst an educated guess should be used. Anecdotal evidence suggests that working out the creatinine clearance for these patients helps with other aspects of prescribing; for example, with the antibiotics vancomycin and gentamicin. Both of these antibiotics need creatinine clearances to guide dosing. It would appear on talking to medical staff that these antibiotics take precedent when working out creatinine clearance as if they are somewhat more 'important' or 'dangerous' and therefore always need prescribed correctly. It was only when we were able to show the data on iatrogenic haemorrhage and the low compliance with correct prescribing that staff was then able to take on board the dangers enoxaparin.

\section{Conclusion}

This audit was carried out in the orthopaedics department of the Royal Alexandra Hospital in Paisley. It was set up in response to continued documentation of prescribing errors, especially in thrombo prophylaxis. Our audit concerned itself with new prescribing data showing that higher doses of clexane in the long term can lead to increased bleeding risks. We were concerned that elderly patients, with poor renal function and low body weight were being subjected to these increased risks and therefore at an increased morbidity risk. It is important to point out that there is no effective way to reverse the action of enoxaparin, or indeed any low molecular weight heparin. It is for this reasons that we implemented a full audit cycle to assess and improve junior doctor prescribing habits. The first audit cycle showed an error rate of $11 \%$ which was much higher than anticipated. There are nearly 100 new admissions a week within the department and this would have accounted for more than 500 prescribing errors a year. Our intervention addressed firstly educating the current junior staff by increasing their awareness of the dangers of enoxaparin. Furthermore, posters and presentations helped to promote good prescribing practice. The re-audit showed a reduction in error to $4 \%$. This was a simple audit, carried out effectively with proof of a change in current practice. The template exists within the department to assess compliance in future years. Further research could follow up cohorts that received an incorrect dose to document whether there was an increase in morbidity or mortality.

\section{References}

1. British National Formulary, BMJ Group, September 2012, Appendixes.

2. Electronic Medicines Compendium, Website accessed 20/01/2013 